Mappemonde

Revue trimestrielle sur l'image géographique et les

formes du territoire

$120 \mid 2017$

Varia

\title{
Magrit : ceci est un logiciel de cartographie
}

\section{Hadrien Commenges}

URL : http://journals.openedition.org/mappemonde/3087

DOI : 10.4000/mappemonde.3087

ISSN : 1769-7298

Éditeur

UMR ESPACE

\section{Référence électronique}

Hadrien Commenges, « Magrit : ceci est un logiciel de cartographie », Mappemonde [En ligne], 120 |

2017, mis en ligne le 01 avril 2017, consulté le 14 septembre 2020. URL : http://

journals.openedition.org/mappemonde/3087

Ce document a été généré automatiquement le 14 septembre 2020.

\section{(c) (i) (2)(2)}

La revue Mappemonde est mise à disposition selon les termes de la Licence Creative Commons Attribution - Pas d'Utilisation Commerciale - Partage dans les Mêmes Conditions 4.0 International. 


\section{Magrit : ceci est un logiciel de cartographie}

\section{Hadrien Commenges}

Le Réseau interdisciplinaire de recherche sur l'aménagement et la cohésion des territoires de l'Europe et de ses voisinages, ou UMS RIATE, développe depuis plusieurs années un ensemble d'outils géomatiques, en particulier des packages pour le logiciel $\mathrm{R}$ (SpatialPosition, cartography) et des atlas interactifs (AIRE, HyperAtlas). Magrit (http://magrit.cnrs.fr) est le tout nouvel outil de l'UMS RIATE, développé par Matthieu Viry, Timothée Giraud et Nicolas Lambert. Il s'agit d'un logiciel en ligne de cartographie thématique.

Figure 1. MAGRIT, un logiciel de cartographie aux nombreuses fonctionnalités

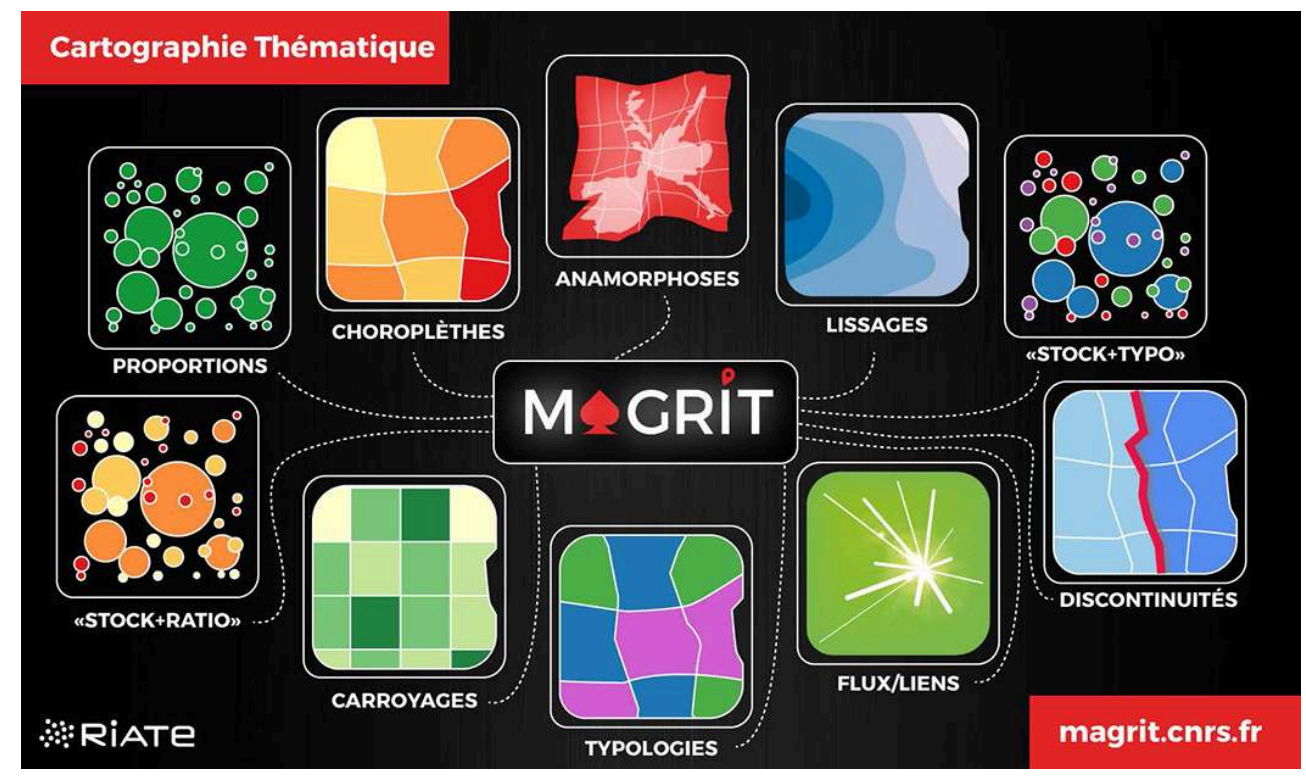




\section{Magrit en bref}

2 Voici en quelques points tout le bien que l'on peut dire de Magrit :

- il s'agit d'un logiciel en ligne qui peut être utilisé sans aucune installation locale. Une connexion Internet et un navigateur suFfisent ;

- l'interface graphique est bien conçue rendant l'utilisation facile et instinctive (user-friendly) ;

- il propose toutes les fonctionnalités essentielles pour un logiciel de cartographie: représentation choroplète, symboles proportionnels, et représentation typologique ;

- il propose des fonctionnalités avancées plus rares: représentations cartographiques bivariées et carroyages ;

- il propose des fonctionnalités introuvables dans une interface intégrée de cartographie: lissage, cartographie des discontinuités, anamorphoses ;

- il lit les principaux formats de données spatiales (Shapefile, GeoJSON, TopoJSON, kml, gml, csv); il gère les systèmes de projection ;

- il permet une mise en forme avancée de la carte avec le paramétrage de nombreux éléments : légende, titre, échelle, Nord ;

- il comporte tous les paramètres nécessaires pour exporter les cartes avec le format de fichier (raster ou vectoriel) et la résolution voulue ;

- il est très bien documenté, avec un blog qui comporte des tutoriels vidéo et une documentation écrite baptisée les Docs de Magrit en référence à la documentation du logiciel Philcarto : les Docs de GRANIT.

Figure 2. Exemple de réalisation de carte bivariée

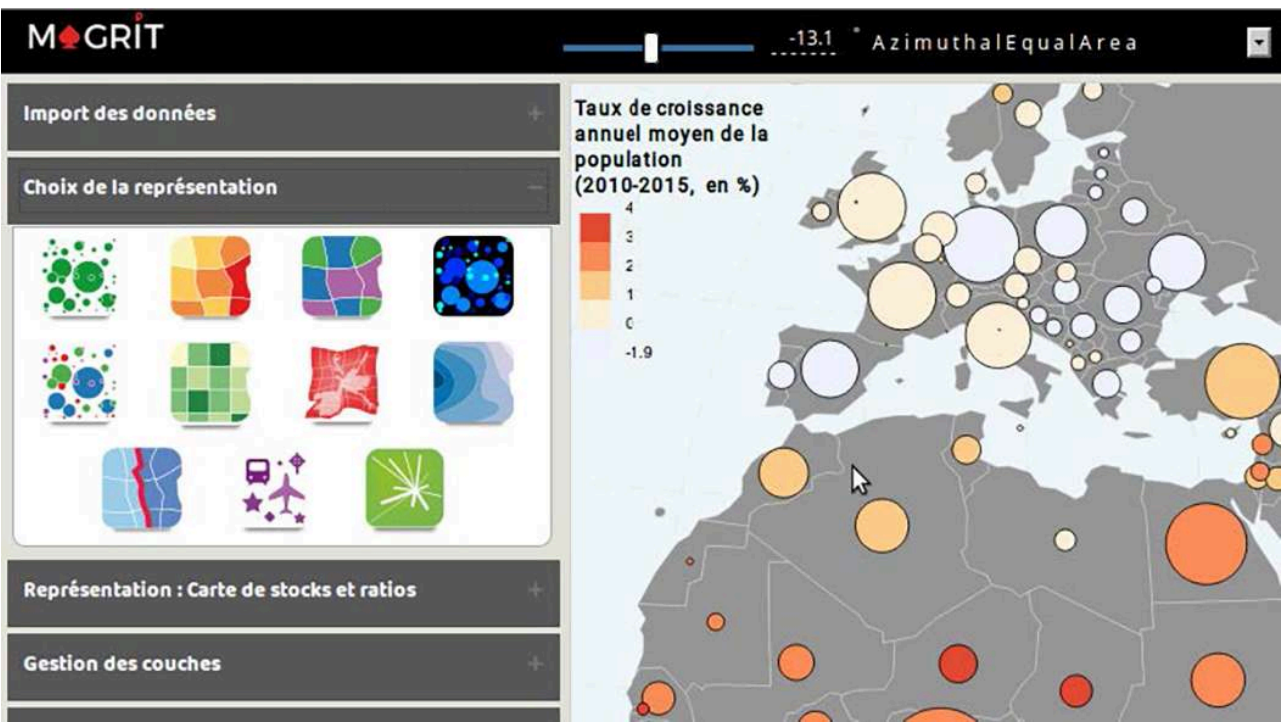

3 À partir de cette liste d'éléments, d'un coup d'œil jeté sur la galerie et des tests efFectués avec mes données, je serais tenté d'afFIrmer que Magrit est le meilleur outil existant actuellement pour faire de la cartographie thématique. Mais cette affirmation est incomplète : pour mieux évaluer l'apport de Magrit, il faut replacer l'outil dans des usages et dans les évolutions récentes du développement logiciel. Dans les deux cas, la comparaison entre Magrit et Philcarto sera fréquente puisque les besoins auxquels répondent les deux logiciels se chevauchent en partie. 


\section{Magrit et ses usages}

4 «L'outil n'est réellement que dans le geste qui le rend techniquement efficace » (LeroiGourhan, 1965). Voici l'une des phrases emblématiques de l'anthropologie des techniques qui s'applique aussi bien à la charrue qu'au logiciel informatique. Un objet technique, comme un logiciel, ne peut pas être analysé comme simple dispositif indépendamment de ses usages (Akrich, 1987). Pour quels usages Magrit est-il un outil particulièrement intéressant?

5 En premier lieu pour l'enseignement. Magrit est installé sur un serveur accessible depuis n'importe quel poste disposant d'un navigateur et d'une connexion Internet. Il ne demande pas d'installation locale, ce qui est un avantage crucial pour qui connait les difficultés à disposer de salles informatiques bien équipées dans les universités et autres établissements d'enseignement. Dans sa conception, le logiciel répond bien à plusieurs objectifs pédagogiques des premières années de licence en géographie. Il faut souligner en particulier la logique de couches qui renvoie aux autres types de logiciels utilisés dans ce domaine : logiciels de SIG (QGIS, ArcGIS), logiciels de dessin vectoriel (Inkscape, Illustrator). Notons également la qualité de l'interface permettant de paramétrer une discrétisation en fonction de la distribution de la variable à discrétiser. Une lacune cependant, le mode de discrétisation autour de la moyenne et de l'écarttype n'est pas implémenté.

6 Ensuite, Magrit est particulièrement indiqué pour la production cartographique « autonome » (standalone), c'est-à-dire pour l'utilisateur qui dispose de données brutes (fond de carte, tableau de données) en entrée et qui veut produire une carte en sortie, sans analyses intermédiaires. Cette remarque porte sur la façon dont la cartographie s'insère dans un flux de travail: l'utilisateur qui souhaite explorer graphiquement et cartographiquement des résultats d'analyses de données (modélisation et fouille de données) trouvera avantageux d'utiliser des logiciels qui intègrent des fonctionnalités d'analyse. C'est sur ce créneau que le logiciel Philcarto s'est illustré, et c'est cette possibilité de coupler analyse de données et cartographie qui explique son succès durable. L'utilisateur qui souhaite faire de l'analyse exploratoire intensive, avec des vaet-vient fréquents entre analyse, manipulation des données et visualisations cartographiques, trouvera avantageux d'utiliser un langage de programmation. Le logiciel-langage R est alors tout indiqué (Groupe ElementR, 2014).

\section{Magrit et la production logicielle}

7 Magrit s'inscrit dans une évolution récente du modèle économique de la production logicielle désignée sous le nom de "software as a service " (SaaS). Le logiciel n'est pas installé sur une machine locale mais déployé sur un serveur distant auquel l'utilisateur accède par une connexion Internet. Pour les logiciels commerciaux, ce nouveau modèle économique présente plusieurs avantages, en particulier (1) le paiement régulier du client par abonnement, (2) la possibilité d'évaluer systématiquement l'utilisation qui est faite du logiciel,(3) la facilité de déploiement sans avoir à prévoir toutes les configurations locales des machines des utilisateurs. L'UMS RIATE n'étant pas une entreprise à but lucratif mais une unité de service du CNRS, le choix d'un logicielservice (SaaS) est surtout dicté par le point $\mathrm{n}^{\circ} 3$. Ce choix a des implications importantes pour l'utilisateur : Magrit est utilisable sans installation spécifique et quel 
que soit le système opérationnel de l'utilisateur. C'est un énorme avantage vis-à-vis de Philcarto, par exemple, qui nécessite un système Windows.

8 L'évolution vers des logiciels-service pose la question de la protection des données. Qu'advient-il des données lorsqu'elles sont chargées sur l'application? Dans le cas de Magrit, il y a un engagement à détruire les données dès que l'utilisateur ferme la session, mais c'est bien sûr une question de confiance de l'utilisateur envers les gestionnaires du serveur distant. Dans un grand nombre de cas, en enseignement en particulier, les données ne nécessitent pas de protection particulière (données libres, issues de l'Insee, de l'IGN, etc.) et le problème est mineur. Si l'utilisateur désire utiliser le logiciel avec des données sensibles, il reste possible de déployer le logiciel sur son propre serveur ou sur sa propre machine (voir ci-dessous), mais cette possibilité demande des compétences informatiques plus avancées.

Enfin, Magrit est un logiciel libre sous licence CeCILL qui respecte les libertés logicielles fondamentales : utiliser le programme, le copier, avoir accès au code source et pouvoir l'analyser, le modifier et le diffuser. Ce choix marque aussi une différence importante vis-à-vis de Philcarto. Dans un retour historique sur le développement de ce logiciel, Philippe Waniez avait écrit à propos du libre : «ce serait une excellente chose si, dans notre pays, le risque de plagiat n'était pas si grand » (Waniez, 2010). Ainsi, Philcarto est un logiciel gratuit mais non libre, son code n'étant pas accessible. Magrit en revanche s'appuie sur un ensemble de technologies et de formats libres. L'utilisateur peut examiner son code, le diffuser, il peut contribuer à son développement. Il peut aussi déployer le logiciel localement ou sur un autre serveur, opération facilitée par l'utilisation de Docker qui permet le déploiement dans des conteneurs logiciels. Là encore, Magrit est à la pointe de la technologie actuelle, Docker étant un logiciel récent (2013) en pleine expansion.

Le choix de développer un logiciel propriétaire ou un logiciel libre a des implications très pragmatiques pour les utilisateurs, en particulier dans un contexte d'enseignement. Mais il s'agit in fine d'un choix politique et culturel : le libre véhicule "un ensemble de valeurs et de comportements", en particulier "les principes de partage de connaissances et de biens (culturels, artistiques, techniques, etc.), les modèles d'organisation et d'économie basés sur ces principes, les valeurs culturelles et sociales ainsi que les comportements résultants de l'adhésion à ces modèles et ces valeurs. » (Paloque-Berges et Masutti, 2013). Les personnels de l'enseignement et de la recherche produisent des objets qui participent à la création et à la diffusion des connaissances : des données, des logiciels, des articles, des livres. Si on accepte l'idée qu'il y a une cumulativité des connaissances (Pumain, 2005), il est évident que leur mode de production et de diffusion est au cœur de cette dynamique et donc au cœur de notre métier. L'option libre a fait ses preuves dans le domaine des SIG avec QGIS et PostGIS, elle a fait ses preuves dans le domaine de l'analyse de données et l'analyse spatiale avec R. Magrit est une nouvelle pierre apportée à l'édifice pour la cartographie. 


\section{BIBLIOGRAPHIE}

AKRICH M. (1987). « Comment décrire les objets techniques ? ». Techniques et culture, vol. 9, p. 49-64.

GROUPE ELEMENTR (2014). R et espace. Traitement de l'information géographique. Framabook. https:// framabook.org/r-et-espace

LEROI-GOURHAN A. (1965). Le geste et la parole. La mémoire et les rythmes, tome 2. Paris : Albin Michel, $288 \mathrm{p}$.

PALOQUE-BERGES C., MASUTTI Ch. (2013). Histoires et cultures du Libre. Des logiciels partagés aux licences échangées. Framabook. https://framabook.org/histoiresetculturesdulibre

PUMAIN D. (2005). «Cumulativité des connaissances ». Revue européenne des sciences sociales, XLIII-131, p. 5-12.

WANIEZ Ph. (2010). «Philcarto : histoire de vie d'un logiciel de cartographie ». Cybergeo : European Journal of Geography, Cartographie, Imagerie, SIG, document 497, mis en ligne le 03 mai 2010, consulté le 21 mars 2017. https://journals.openedition.org/cybergeo/23076

\section{AUTEUR}

HADRIEN COMMENGES

Université Paris 1 Panthéon-Sorbonne, UMR Géographie-cités 